\title{
ANÁLISE DA CURVA DE CRESCIMENTO DE MACHOS HEREFORD ${ }^{1}$
}

\author{
ANA RITA DE ASSUMPÇÃO MAZZINI ${ }^{2}$ \\ JOEL AUGUSTO MUNIZ ${ }^{3}$ \\ LUIZ HENRIQUE DE AQUINO ${ }^{4}$ \\ FABYANO FONSECA E SILVA
}

\begin{abstract}
RESUMO - Para estudar a função de melhor ajustamento ao crescimento de bovinos Hereford, foram utilizados dados de 160 machos, ajustando-se as funções de Brody, Gompertz, Logística, Richards e von Bertalanffy. A estimação dos parâmetros para modelos de regressão não-linear foi feita pelo método dos Quadrados Mínimos Ponderado. Para isso, utilizou-se o procedimento MODEL do software Statistical Analysis System (SAS). Foram obtidos ajustes de curvas individuais para os animais em dois diferentes modelos: não-ponderado e ponderado pelo inverso da variância dos pesos, com o objetivo de levar em consideração a heterogeneidade
\end{abstract}

das variâncias. A comparação entre os modelos foi feita pela interpretação biológica dos parâmetros e dos avaliadores da qualidade de ajuste (coeficiente de determinação ajustado, quadrado médio do resíduo, erro de predição médio, teste t de Student, para as estimativas dos parâmetros "A" e "K" e percentual de convergência). O melhor modelo foi o ponderado pelo inverso da variância dos pesos. As funções que apresentaram melhor ajuste foram as de von Bertalanffy e Gompertz, seguidas pela Logística. As funções de Brody e Richards apresentaram um baixo percentual de convergência, mostrando-se inadequadas para descrever os dados em estudo.

TERMOS PARA INDEXAÇÃO: Regressão não-linear, heterogeneidade de variâncias, curva de crescimento.

\section{GROWTH CURVE ANALYSIS FOR HEREFORD CATTLE MALES}

\begin{abstract}
A weight-age growth curve was studied in 160 bovine Hereford males. It was fitted to the Brody, Gompertz, Logistic, Richards and von Bertalanffy functions. The parameters estimates for the nonlinear regression model were calculated by the Weighted-Least-Square through MODEL procedure (SAS/ETS, 1995). An animal individual adjustment was obtained by the two models: non-weighted and weighted by the inverse of variance. The comparison with the previous models was made by the parameters
\end{abstract}

biological interpretations and by fit quality evaluators (Adjusted determination coefficient, error mean square, mean predicted error, Student's t-test for the parameters $\mathrm{A}$ and $\mathrm{K}$ estimates and convergency percent). The best fitted model was the weighted by the inverse of variance. The functions that best fitted the data were those of von Bertalanffy and Gompertz followed by Logistic. Brody and Richards functions presented a low convergency percent, being inadequate to describe the data in study.

INDEX TERMS: Nonlinear regression, heterogeneity of variance, growth curve.

\section{INTRODUÇÃO}

Com base em pesquisas que enfocam as fases iniciais de crescimento, a raça Hereford tem tido um merecido destaque no que se refere à precocidade dos animais (ASSOCIAÇÃ̃ O.., 2000). Em um sistema de produção de carne, o crescimento é uma função primordial, pois apresenta relação direta com a quantidade e qualidade da carne, produto final da exploração.

1. Parte da dissertação apresentada à UNIVERSIDADE FEDERAL DE LAVRAS/UFLA - Caixa Postal 37 37200-000 - Lavras, MG, pela primeira autora, para obtenção do grau de Mestre em Agronomia, Área de Concentração em Estatística e Experimentação Agropecuária.

2. Engenheiro Agrônomo, Mestre em Estatística e Experimentação Agropecuária/UFLA, itaalves@terra.com.br

3. Engenheiro Agrônomo, Professor Titular do Departamento de Ciências Exatas/UFLA, Bolsista do CNPq, joamuniz@ufla.br

4. Engenheiro Agrônomo, Professor Titular do Departamento de Ciências Exatas/UFLA, Ihaquino@ufla.br

5. Acadêmico do $10^{\circ}$ Período do Curso de Zootecnia/UFLA, bolsista do PIBIC/CNPq. 
Os órgãos ou tecidos apresentam diferentes taxas e velocidades de crescimento e maturação, influenciados pelo nível nutricional, ocorrendo por ordem prioritária o crescimento do tecido nervoso, ósseo, muscular e adiposo. O aumento do peso de um animal reflete o seu crescimento e ocorre por retenção de água, proteína, gordura e minerais em quantidades variáveis. É uma função básica da alimentação que o animal recebe, das condições climáticas em que se encontra, de seu estado sanitário e das características inerentes à sua genética, biótipo, raça, sexo, peso, idade e estado corporal. A representação gráfica do peso ou massa corporal em relação à idade resulta na curva de crescimento (GOTTSCHALL, 1999).

$\mathrm{Na}$ análise de dados de crescimento, a utilização de funções matemáticas não-lineares, desenvolvidas empiricamente para relacionar peso-idade, tem-se mostrado adequada para descrever a curva de crescimento, uma vez que essas funções sintetizam um grande número de medidas em apenas alguns parâmetros com significado biológico, facilitando, dessa forma, a interpretação e o entendimento do fenômeno (BROWN et al., 1976; DENISE e BRINKS, 1985). Cinco dessas funções têm sido mais utilizadas em estudos de crescimento em bovinos Brody, Gompertz, Logística, Richards e von Bertalanffy. Vários autores já as utilizaram para descrever o crescimento tanto de raças taurinas quanto zebuínas (BROWN et al., 1972a, 1972b; DUARTE, 1975; SILVEIRA JUNIOR, 1976; BROWN et al., 1976; DENISE e BRINKS, 1985; LUDWIG et al., 1981; NOBRE et al., 1987; TEDESCHI, 1996; ELIAS, 1998; OLIVEIRA et al., 2000). Apesar da existência de trabalhos com as diversas raças bovinas, não há relatos de estudos de curvas de crescimento que utilizam dados de pesagens de machos Hereford criados no Brasil.

A maioria dos trabalhos que compara essas funções, em geral, não leva em consideração a relação direta entre a variância dos pesos corporais e a idade dos animais. Pasternak e Shalev (1994) afirmam que o simples ajuste de uma regressão não-linear a dados longitudinais pode ser ineficiente, uma vez que não considera a heterogeneidade de variâncias, o que os autores definiram como "distúrbios de regressão". Elias (1998), em estudo de curvas de crescimento com raças zebuínas, comparou as funções de Brody, Gompertz, Logística, Richards e von Bertalanffy, ajustadas de forma nãoponderada e ponderada, pelo inverso da variância dos pesos, verificando que a utilização da ponderação promoveu uma marcante redução na variabilidade das estimativas dos parâmetros.
Com este trabalho objetivou-se comparar as cinco funções de crescimento mais utilizadas para descrever as relações peso-idade dos animais, considerando-se os modelos não-ponderado e ponderado pelo inverso da variância dos pesos, realizando-se ajustes de curvas individuais para machos da raça Hereford.

\section{MATERIAL E MÉTODOS}

Os dados utilizados neste trabalho provêm de machos da raça Hereford, não castrados, nascidos nos anos de 1992 a 1994 na Agropecuária Recreio, situada no município de Bagé, Rio Grande do Sul e foram cedidos pelo Centro de Pesquisas Pecuária Sul, pertencente à Empresa Brasileira de Pesquisa Agropecuária (EMBRAPA).

O manejo alimentar constituiu-se de pasto nativo, pastagem cultivada e suplementação a campo (do desmame até o $1^{\circ}$ ano).

As pesagens consideradas no estudo foram coletadas de 160 animais, desde o nascimento até aproximadamente 790 dias de idade, obtendo-se no grupo 47 animais com 16 pesagens; 30 animais com 17 pesagens; 24 animais com 18 pesagens; 51 animais com 19 pesagens e 8 animais com 20 pesagens.

O método de estimação de parâmetros foi o dos Quadrados Mínimos Ponderado, implementado pelo procedimento MODEL do SAS ${ }^{\circledR}$ (SAS, 1995), o qual emprega os processos iterativos de Gauss Newton e Marquardt. Foram obtidas estimativas médias para as curvas individuais nos modelos não-ponderados e ponderados pelo inverso das variâncias dos pesos em cada pesagem, calculadas pelo procedimento UNIVARIATE do SAS. As estimativas dos pesos preditos foram comparadas com os estabelecidos como valores médios da raça, fornecidos pela ASSOCIAÇÃO NACIONAL DE CRIADORES-HERD BOOK COLLARES (2000) e ASSOCIAÇÃO... (2000).

Para executar a ponderação, foi calculada para cada intervalo de tempo, de $\mathrm{T}_{1}$ (nascimento) até $\mathrm{T}_{20}$ (idade aos 790 dias), a variância dos pesos. O inverso dessas variâncias foi utilizado como fator de ponderação pela opção WEIGHT do procedimento MODEL (SAS, 1995).

As funções foram comparadas quanto ao percentual de convergência, quadrado médio do resíduo (QMR), coeficiente de determinação ajustado $\left(\mathrm{R}^{2} \mathrm{Aj}\right.$.), erro de predição médio (EPM) e teste $t$ de Student.

$\mathrm{O}$ coeficiente de determinação ajustado para o número de parâmetros da regressão dado por, 
$\mathrm{R}_{\text {Ajustado }}^{2}=1-\left[\frac{(\mathrm{n}-\mathrm{i})\left(1-\mathrm{R}^{2}\right)}{(\mathrm{n}-\mathrm{p})}\right]$, em que: $\mathrm{R}^{2}$ - é o

coeficiente de determinação; $n$ - número de observações utilizadas para ajustar a curva; $\mathrm{p}$ - número de parâmetros na função, incluindo o intercepto; i - ajuste de intercepto: 1 , se houver intercepto e 0 , se não houver intercepto na função (SAS, 1995).

A estimativa do EPM foi obtida calculando-se a média de todos os erros de predição (EP), considerando-se cada observação como um desvio entre o peso observado (po) e o peso estimado (pe), dividido pelo peso observado e multiplicado por 100, ou seja, EP $=100\left(\frac{\text { po }- \text { pe }}{\text { po }}\right)$.

Este valor leva um sinal (+) ou (-), designando se a função subestimou ou superestimou, respectivamente, o peso observado (GOONEWARDENE et al., 1981).
Para julgar a hipótese de que os parâmetros A, b, $\mathrm{K}$ e $\mathrm{M}$ dos modelos são iguais a zero $(\theta=0)$, contra a hipótese de serem diferentes de zero $(\theta \neq 0)$, utilizouse o teste t de Student, pois, de acordo com Ratkowsky (1983), esse é um critério útil para avaliar o comportamento de um modelo não-linear. $\mathrm{O}$ teste é dado por: $\mathrm{t}=\frac{\hat{\theta}}{\mathrm{s}(\hat{\theta})}$, em que o valor de " $\mathrm{t}$ " é a razão entre a estimativa do parâmetro e seu erro padrão, obtido a partir da raiz quadrada da variância assintótica do parâmetro.

\section{RESULTADOS E DISCUSSÃO}

Na Tabela 1, constam os valores dos pesos médios dos animais, suas variâncias em cada idade e o fator de ponderação.

TABELA 1 - Valores médios dos pesos observados, respectivas variâncias no decorrer do tempo e inverso dessas variâncias (fator de ponderação).

\begin{tabular}{|c|c|c|c|c|c|}
\hline Pesagens & $\begin{array}{l}\text { Idade } \\
\text { (dias) }\end{array}$ & № de animais & Peso & $\begin{array}{l}\text { Variância } \\
\left(\mathrm{kg}^{2}\right)\end{array}$ & $\begin{array}{l}\text { Fator de Ponderação } \\
\qquad\left(\mathrm{kg}^{-2} \times 10^{-4}\right)\end{array}$ \\
\hline $\mathrm{P} 1$ & 0 & 160 & 35 & 13,95 & 716,8459 \\
\hline $\mathrm{P} 2$ & 168 & 160 & 189,97 & 3105,72 & 3,219865 \\
\hline P3 & 214 & 160 & 213,76 & 3037,99 & 3,29165 \\
\hline P4 & 243 & 160 & 243,64 & 2383,03 & 4,196338 \\
\hline P5 & 275 & 160 & 281,12 & 2657,6 & 3,762793 \\
\hline P6 & 311 & 160 & 324,39 & 2704,35 & 3,697746 \\
\hline $\mathrm{P} 7$ & 344 & 160 & 363,9 & 3372,86 & 2,964843 \\
\hline P8 & 377 & 160 & 404,22 & 3238,55 & 3,087802 \\
\hline P9 & 414 & 160 & 443,92 & 3468,11 & 2,883415 \\
\hline P10 & 472 & 160 & 489,51 & 4722,59 & 2,117482 \\
\hline P11 & 508 & 160 & 518,54 & 6041,73 & 1,655155 \\
\hline P12 & 542 & 160 & 541,34 & 5853,09 & 1,708499 \\
\hline P13 & 594 & 160 & 559,21 & 5788,24 & 1,727641 \\
\hline P14 & 624 & 160 & 583,81 & 6578,37 & 1,520133 \\
\hline P15 & 660 & 160 & 611,76 & 6542,95 & 1,528363 \\
\hline P16 & 692 & 160 & 639,24 & 8667,74 & 1,153703 \\
\hline P17 & 710 & 113 & 674,59 & 10026,15 & 0,997392 \\
\hline P18 & 720 & 83 & 693,12 & 8833,11 & 1,132104 \\
\hline P19 & 742 & 56 & 718,15 & 2493,03 & 4,011183 \\
\hline P20 & 795 & 8 & 727,12 & 1546,7 & 6,465378 \\
\hline
\end{tabular}


Pode-se observar que à medida que a idade aumentou, houve um incremento nas variâncias dos pesos corporais. Esses resultados estão de acordo com Pasternak e Shalev (1994), os quais observaram que a variância dos pesos corporais aumenta com a idade, ocorrendo a heterocedasticidade. Elias (1998) também observou um aumento da variância dos pesos ao longo do tempo para as raças Gir, Guzerá e Nelore.

Na Tabela 2 estão apresentados os percentuais de convergência, para as cinco funções estudadas, considerando os modelos não-ponderados e ponderados.

TABELA 2 - Percentual de convergência para as cinco funções nos dois modelos ajustados.

\begin{tabular}{lcc}
\hline \multicolumn{1}{c}{ Funções } & $\begin{array}{c}\text { Modelo } \\
\text { não-ponderado }\end{array}$ & $\begin{array}{c}\text { Modelo } \\
\text { ponderado }\end{array}$ \\
\hline Brody & 23,13 & 6,87 \\
Gompertz & 92,50 & 95,00 \\
Logístico & 100,00 & 100,00 \\
Richards & 46,25 & 41,88 \\
von Bertalanffy & 79,38 & 87,50 \\
\hline
\end{tabular}

Percebe-se, de modo geral, para as duas configurações de modelos estudadas, que as funções Logística, Gompertz e von Bertalanffy, respectivamente, apresentaram maiores percentuais de convergência. Esse resultado está de acordo com Oliveira et al. (2000), os quais, em trabalho com curvas de crescimento de fêmeas Guzerá, obtiveram maiores percentuais de convergência para essas funções e menores para as de Brody e Richards. Observou-se ainda uma certa tendência no aumento do percentual de convergência, nas funções de Gompertz e von Bertalanffy, quando se utilizou a ponderação. Esse resultado concorda parcialmente com Elias (1998), que observou um aumento da convergência em todas as funções com a utilização da ponderação pelo inverso da variância. As funções de Brody e Richards mostraram baixos percentuais de convergência, inferindo-se que possivelmente não estejam adequadas ao ajuste desses dados. Vários autores alertam sobre a dificuldade de convergência na função de Richards (BROWN et al., 1976; FITZHUGH JUNIOR, 1976; DENISE e BRINKS, 1985; PEROTTO et al., 1992). Já o problema ocorrido na função de Brody foi que muitos valores convergiram para valores de $\mathrm{A}$ fora do espaço paramétrico, do ponto de vista prático, resultado esse semelhante ao observado por Ludwig et al. (1981), que também observaram convergência dessa função para valores fora da realidade.

Na Tabela 3 são apresentados os quadrados médios dos resíduos para os dois modelos ajustados, nas cinco diferentes funções.

TABELA 3 - Médias do quadrado médio do resíduo (QMR) para as cinco funções, nos dois modelos ajustados.

\begin{tabular}{lcc}
\hline \multicolumn{1}{c}{ Funções } & $\begin{array}{c}\text { Modelo } \\
\text { não-ponderado }\end{array}$ & $\begin{array}{c}\text { Modelo } \\
\text { ponderado }\end{array}$ \\
\hline Brody & 891,62 & 0,3416 \\
Gompertz & 750,16 & 0,2006 \\
Logístico & 960,58 & 0,4066 \\
Richards & 797,48 & 0,1512 \\
Von Bertalanffy & 724,82 & 0,1679 \\
\hline
\end{tabular}

Pode-se observar que, para o modelo nãoponderado, as funções de von Bertalanffy e Gompertz apresentaram um menor QMR. Esse resultado concorda com Oliveira et al. (2000) no que se refere a função de von Bertalanffy, e com Tedeschi (1996), no que diz respeito a função de Gompertz. Para o modelo ponderado, as funções de menor QMR foram as de Richards, von Bertalanffy e Gompertz, de forma semelhante aos resultados encontrados por Elias (1998), no que se refere às funções de Richards e von Bertalanffy.

Na Tabela 4 são mostradas as médias dos coeficientes de determinação ajustados, tanto para os modelos não-ponderados quanto para os ponderados. Observa-se por meio de seus valores um bom ajuste para todas as funções; entretanto, nota-se uma tendência de valores maiores de $\mathrm{R}^{2} \mathrm{Aj}$. nos modelos ponderados.

TABELA 4 - Médias dos coeficientes de determinação ajustados $\left(R^{2} \mathrm{Aj}\right.$.) para as cinco funções, nos dois modelos ajustados.

\begin{tabular}{lcc}
\hline \multicolumn{1}{c}{ Funções } & $\begin{array}{c}\text { Modelo } \\
\text { não-ponderado }\end{array}$ & $\begin{array}{c}\text { Modelo } \\
\text { ponderado }\end{array}$ \\
\hline Brody & 0,9735 & 0,9939 \\
Gompertz & 0,9774 & 0,9950 \\
Logístico & 0,9719 & 0,9893 \\
Richards & 0,9756 & 0,9958 \\
Von Bertalanffy & 0,9778 & 0,9956 \\
\hline
\end{tabular}


Elias (1998), em análise de curvas de crescimento para vacas zebuínas, encontrou coeficiente de determinação de aproximadamente 0,999 para todas as funções, tanto para os ajustes dos modelos não-ponderados quanto para os modelos ponderados. Ludwig et al. (1981), em estudo com gado Nelore, concluíram que todas as funções ajustaram-se bem aos dados de peso-idade, apresentando coeficientes de determinação próximos de 0,99. Oliveira et al. (2000) concluíram que as diferenças entre os coeficientes de determinação foram irrisórias e sem significado prático. Em última instância, nas condições do presente trabalho, observou-se que esse avaliador não é um bom indicador para escolha da melhor função, já que todas as funções apresentaram valores altos.

$\mathrm{Na}$ Tabela 5 são apresentados os valores médios da estatística t para as estimativas dos parâmetros A e K das funções estudadas nas duas configurações.

TABELA 5 - Teste $\mathrm{t}$ de Student, para os valores paramétricos de $\mathrm{A}$ e $\mathrm{K}$ das funções estudadas, para os modelos não-ponderado e ponderado.

\begin{tabular}{lcccc}
\hline \multirow{2}{*}{ Funções } & \multicolumn{2}{c}{$\begin{array}{c}\text { Modelo } \\
\text { não- } \\
\text { ponderado }\end{array}$} & \multicolumn{2}{c}{$\begin{array}{c}\text { Modelo } \\
\text { ponderado }\end{array}$} \\
\cline { 2 - 6 } & \multicolumn{1}{c}{$\mathbf{A}$} & $\mathbf{K}$ & \multicolumn{1}{c}{$\mathbf{A}$} & $\mathbf{K}$ \\
\hline Brody & 6,64 & 3,90 & 7,97 & 5,46 \\
Gompertz & 14,88 & 7,37 & 24,43 & 20,61 \\
Logística & 20,51 & 8,66 & 28,05 & 24,19 \\
Richards & 5,13 & 1,73 & 6,35 & 2,40 \\
Von Bertalanffy & 12,31 & 6,41 & 19,44 & 16,00 \\
\hline
\end{tabular}

A estatística t para o teste sobre os valores paramétricos de $\mathrm{A}$ e $\mathrm{K}$ das funções estudadas mostra que nos dois modelos, não-ponderado e ponderado, o valor mais alto da estatística t, tanto para o parâmetro A quanto para o parâmetro $K$, foi obtido na função Logística, seguida pelas funções de Gompertz e von Bertalanffy. Também se pode notar que nos modelos ponderados os valores da estatística $t$ foram mais altos do que nos nãoponderados, significando que a ponderação aumenta a probabilidade de os parâmetros serem diferentes de zero.

Na Tabela 6 são mostradas as médias dos EPM's das cinco funções estudadas nos dois modelos diferentes.
TABELA 6 - Médias dos Erros de Predição Médios (EPM) para as cinco funções nos diferentes modelos ajustados

\begin{tabular}{lcc}
\hline \multicolumn{1}{c}{ Funções } & $\begin{array}{c}\text { Modelo } \\
\text { não-ponderado }\end{array}$ & $\begin{array}{c}\text { Modelo } \\
\text { ponderado }\end{array}$ \\
\hline Brody & 1,5774 & $-1,8227$ \\
Gompertz & $-2,5861$ & 0,7283 \\
Logístico & $-6,2442$ & 1,8057 \\
Richards & 0,4348 & $-0,7322$ \\
Von Bertalanffy & $-0,9989$ & 0,2898 \\
\hline
\end{tabular}

Pode-se observar na Tabela 6 que, para o modelo não-ponderado, as funções de Brody e Richards subestimaram o valor observado, enquanto as funções de von Bertalanffy, Gompertz e Logística superestimaram. A função de Richards apresentou a menor média para o erro de predição médio, seguida da função de von Bertalanffy. No caso do modelo ponderado, as funções de Brody e Richards superestimaram os pesos observado, enquanto as demais funções subestimaram, ao contrário do ocorrido no modelo não-ponderado. A menor média observada para o EPM foi na função de von Bertalanffy, seguida pelas funções de Gompertz e Richards. Esse resultado concorda com Elias (1998) no que diz respeito à função de Richards. Também é importante observar que nessa configuração as médias dos EPM's foram mais próximas entre si e, em geral, mais próximas de zero, indicando uma melhora no ajuste por meio da ponderação.

Na Figura 1, estão representadas as curvas de crescimento para as funções de Brody, Gompertz, Logística, Richards e von Bertalanffy, considerando os dois modelos estudados.

Analisando-se a Figura 1, nota-se um melhor ajuste dos dados para todas as funções quando se utilizou a ponderação pelo inverso das variâncias, concordando com os resultados obtidos por Elias (1998), pelos quais infere-se que a melhor função foi a de Brody ponderada, ao contrário do observado no presente trabalho, no qual se constatou que a função de Brody foi a de pior ajuste para os dois modelos. Os melhores ajustes foram obtidos para as funções de von Bertalanffy e Gompertz. Oliveira et al. (2000) e Tedeschi (1996), em estudo com raças zebuínas, consideraram as funções de von Bertalanffy e Gompertz, respectivamente, como as de melhor ajuste aos dados, no caso de modelos não-ponderados.

No ajuste da função Logística, nota-se uma subestimação dos pesos finais, concordando com Brown et 
al. (1976), os quais fizeram as mesmas considerações sobre essa função ajustada a dados de vacas de diversas raças. A função de Richards apresentou um bom ajuste aos dados, sem maiores diferenças entre os dois modelos; porém, vários autores, entre eles Silva et al. (2001), verificaram que essa função, apesar de gerar bons ajustes a dados de crescimento de bovinos, muitas vezes, não é indicada por apresentar baixos percentuais de convergência.

Na Figura 2 estão representados os Box Plot para as estimativas do parâmetro A das cinco funções estudadas no modelo não-ponderado $(\mathrm{A})$ e ponderado $(\mathrm{C}) \mathrm{e}$ do parâmetro K no modelo não-ponderado (B) e ponderado (D).

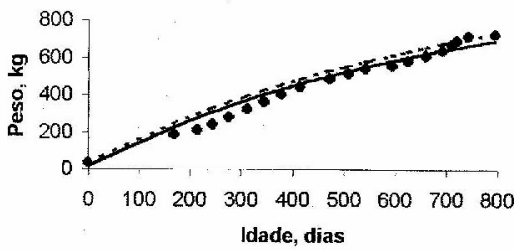

- Observado Modelo não ponderado Modelo ponderado

(A)

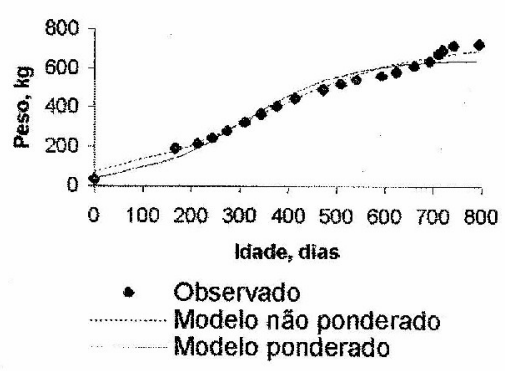

(C)

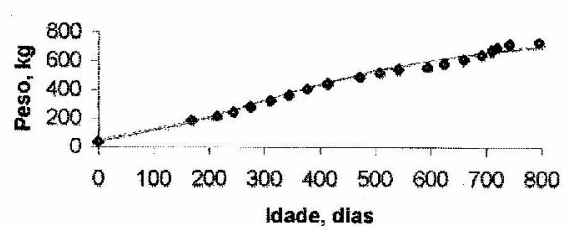

- Observado Modelo não ponderado - Modelo onderado

(B)

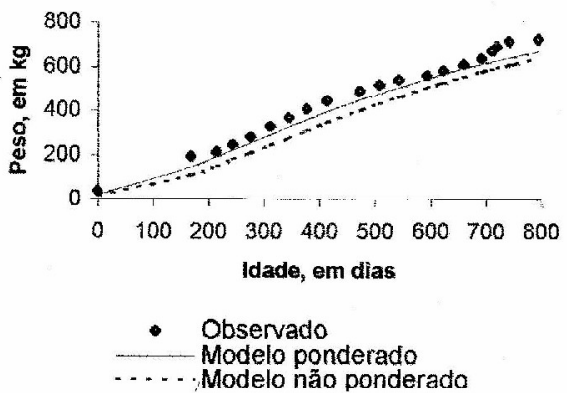

(D)

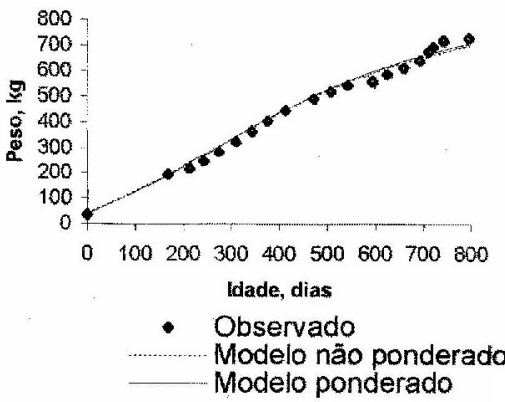

(E)

FIGURA 1 - Ajuste das curvas de crescimento para os modelos não-ponderado e ponderado das funções de Brody (A), Gompertz (B), Logística (C), Richards (D) e von Bertalanffy (E). 


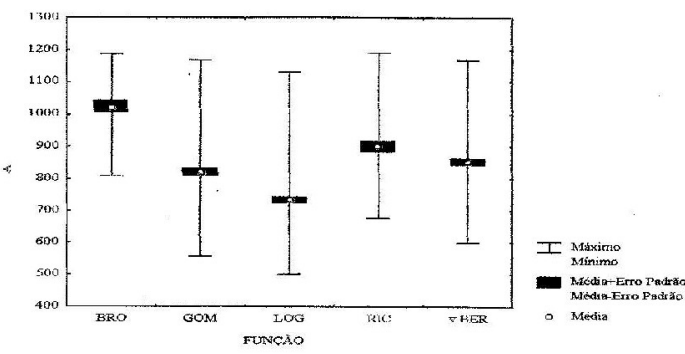

(A)

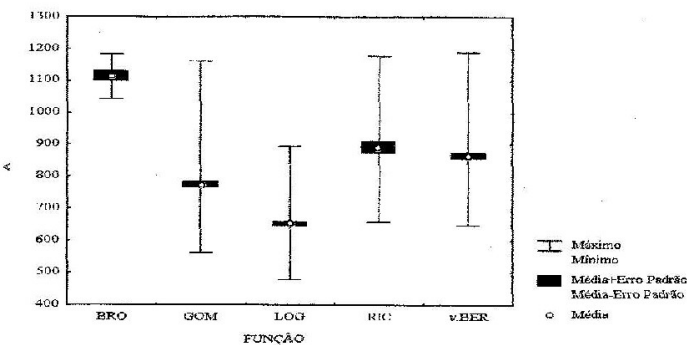

(C)

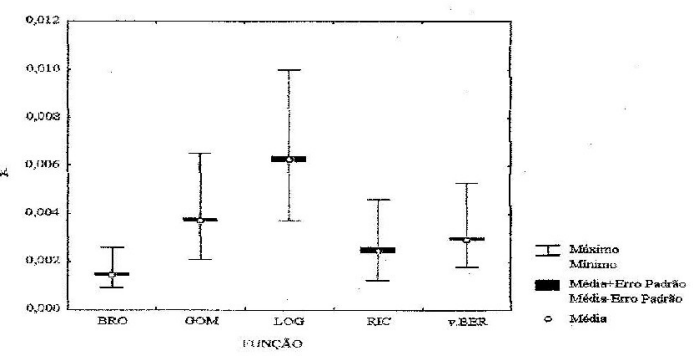

(B)

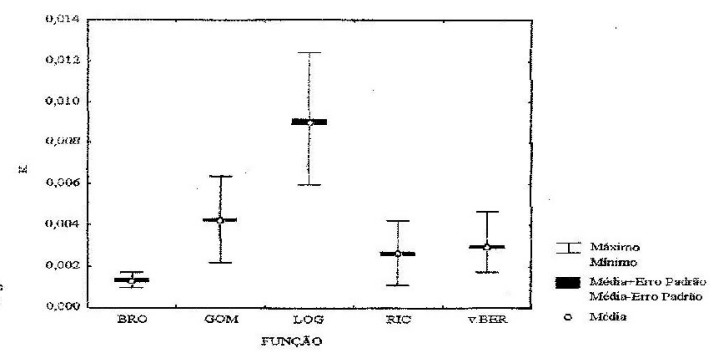

(D)

FIGURA 2 - Box Plot para os modelos não ponderado (A e B) e ponderado (C e D), para as estimativas dos parâmetros A e K.

Na Figura 2 pode ser visualizada, de uma maneira mais clara, a tendência da função Brody de superestimar o peso adulto (parâmetro A) e da função Logística de subestimá-lo nos modelos ponderado e nãoponderado.

Observando-se as Figuras 2A e 2C, pode-se verificar que as funções Brody e Richards apresentam uma maior variabilidade das estimativas do parâmetro A, em relação às funções de Gompertz, Logística e von Bertalanffy. Na Figura 2B, nota-se que o parâmetro K apresentou baixa variabilidade entre suas estimativas para todas as funções, sendo um pouco maior na função Logística e de Richards. Nota-se que, na Figura 2D, a maior variabilidade do parâmetro $\mathrm{K}$ foi para a função Logística, que apresentou também um maior valor médio para essa estimativa.

\section{CONCLUSÕES}

Nas condições do presente estudo e de acordo com os resultados obtidos, pode-se concluir que:

a) De forma geral, considerando todas as funções estudadas, a ponderação pelo inverso das variâncias dos pesos melhorou a qualidade do ajuste; b) As funções de von Bertalanffy e Gompertz foram as que melhor se ajustaram aos dados;

c) As funções de Brody e Richards foram as menos indicadas, pelo seu pior desempenho;

d) A função Logística, embora tenha mostrado um bom ajuste aos dados, tendeu a subestimar o peso adulto.

\section{REFERÊNCIAS BIBLIOGRÁFICAS}

ASSOCIAÇÃO Brasileira de Hereford e Braford. Disponível em: $i<\mathrm{http} / / / \mathrm{www}$.hereford.com.br/ind $2 . \mathrm{htm}>$. Acesso em: 7 nov. 2000 .

ASSOCIAÇÃO NACIONAL DE CRIADORES-HERD BOOK COLLARES; ASSOCIAÇÃO BRASILEIRA DE CRIADORES DE HEREFORD E BRAFORD. XXI-XXIII Exposição Internacional de Animais: raça Polled Hereford. Pelotas, 2000.

BROWN, J. E.; BROWN, C. J.; BUTTS, W. T. A discussion of the genetic aspects of weight, mature weight and rate of maturing in Hereford and Angus cattle. Journal of Animal Science, Champaign, v. 34, n. 4, p. 525-537, Apr. 1972a. 
BROWN, J. E.; BROWN, C. J.; BUTTS, W. T. Relationships among weights, gains and earliness of maturing in Hereford and Angus females. Journal of Animal Science, Champaign, v. 35, n. 3, p. 507-517, Apr. 1972b.

BROWN, J. E.; FITZHUGH JUNIOR, H. A.; CARTWRIGHT, T. C. A comparison of nonlinear models for describing weight-age relationships in cattle. Journal of Animal Science, Champaign, v. 42, n. 4, p. 810-818, Apr. 1976.

DENISE, R. S. K.; BRINKS, J. S. Genetic and environmental aspects of the growth curve parameters in beef cows. Journal of Animal Science, Champaign, v. 61, n. 6, p. 1431-1440, July 1985.

DUARTE, F. A. M. Estudo da curva de crescimento de animais da Raça "Nelore" (Bos taurus indicus) através de cinco modelos estocásticos. 1975. $284 \mathrm{f}$. Tese (Livre Docência em Genética e Matemática aplicada à Biologia) - Universidade de São Paulo, Ribeirão Preto, 1975.

ELIAS, A. M. Análise de curvas de crescimento de vacas das Raças Nelore, Guzerá e Gir. 1998. 128 f. Dissertação (Mestrado em Ciência Animal e Pastagens) - Escola Superior de Agricultura Luiz de Queiroz, Piracicaba, 1998.

FITZHUGH JUNIOR, H. A. Analysis of growth curves and strategies for altering their shapes. Journal of Animal Science, Champaign, v. 42, n. 4, p. 1036-1051, Apr. 1976.

GOONEWARDENE, L. A.; BERG, R. T.; HARDIN, R. T. A growth study of beef cattle. Canadian Journal of Animal Science, Ottawa, v. 61, n. 4, p. 1041-1048, Dec. 1981.

GOTTSCHALL, C. S. Impacto nutricional na produção de carne-curva de crescimento. In: LOBATO, J. F. P.; BARCELlOS, J. O. J.; KESSLER, A. M. Produção de bovinos de corte. Porto Alegre: EDIPUCRS, 1999. p. 169-192.

LUDWIG, A.; SILVA, M. de A.; OLIVEIRA, L. M. de. Ajustamento de modelos estatísticos exponenciais ao crescimento de gado Nelore. Pesquisa Agropecuária Brasileira, Brasília, v. 16, n. 2, p. 297-302, mar. 1981.
NOBRE, P. R. C.; ROSA A. do N.; SILVA, L. O. C. da; EVANGELISTA, S. R. M. Curvas de crescimento de gado Nelore ajustadas para diferentes freqüências de pesagens. Pesquisa Agropecuária Brasileira, Brasília, v. 22, n. 9/10, p. 1027-1037, set./out. 1987.

OLIVEIRA, H. N. de; LÔBO, R. B.; PEREIRA, C. S. Comparação de modelos não-lineares para descrever o crescimento de fêmeas da raça Guzerá. Pesquisa Agropecuária Brasileira, Brasília, v. 35, n. 9, p. 1843 1851 , set. 2000.

PASTERNAK, H.; SHALEV, B. A. The effect of a feature of regression disturbance on the efficiency of fitting growth curves. Growth, Development \& Aging, Bar Harbor, v. 58, n. 1, p. 33-39, 1994.

PEROTTO, D.; CUE, R. I.; LEE, A. J. Comparison of nonlinear functions for describing the growth curve of three genotypes of dairy cattle. Canadian Journal of Animal Science, Ottawa, v. 72, n. 4, p. 773-782, Dec. 1992.

RATKOWSKY, D. A. Nonlinear regression modeling: a unified practical approach. New York: Marcel Dekker, 1983. v. 48, 276 p.

SAS INSTITUTE. SAS/ETS ${ }^{\circledR}$ User's Guide: version 6 . 2. ed. Cary, 1995.

SILVA, F. F. da; AQUINO, L. H. de; OLIVEIRA, A. I. G. de. Influência de fatores genéticos e ambientais sobre as estimativas dos parâmetros das funções de crescimento em gado Nelore. Ciência e Agrotecnologia, Lavras, 2001. No prelo.

SILVEIRA JÚNIOR, P. Estudos de alguns modelos exponenciais no crescimento de bovinos da Raça Ibagé. 1976. 174 f. Dissertação (Mestrado em Estatística e Experimentação Agronômica) - Escola Superior de Agricultura Luiz de Queiroz, Piracicaba, 1976.

TEDESCHI, L. O. Determinação dos parâmetros da curva de crescimento de animais da Raça Guzerá e seus cruzamentos alimentados a pasto, com e sem suplementação. 1996. 140 f. Dissertação (Mestrado em Ciência Animal e Pastagens) - Escola Superior de Agricultura Luiz de Queiroz, Piracicaba, 1996. 